\title{
Hypofibrinogenemia is associated with a high degree of risk in infectious diseases: a post-hoc analysis of post-marketing surveillance of patients with disseminated intravascular coagulation treated with thrombomodulin alfa
}

Kazuo Kawasugi ${ }^{1}$, Hideo Wada ${ }^{2 *}$, Goichi Honda ${ }^{3}$, Noriaki Kawano $^{4}$, Toshimasa Uchiyama ${ }^{5}$, Seiji Madoiwa ${ }^{6}$, Naoki Takezako ${ }^{7}$, Kei Suzuki ${ }^{8}$, Yoshinobu Seki ${ }^{9}$, Takayuki Ikezoe ${ }^{10}$, Toshiaki Iba $^{11}$ and Kohji Okamoto ${ }^{12}$

\begin{abstract}
Background: In patients with infectious diseases, disseminated intravascular coagulation (DIC) is often diagnosed without the fibrinogen value. The relationship between hypofibrinogenemia and outcomes of DIC in infectious diseases has thus remained unclear.

Methods: We analyzed 3204 patients who received with thrombomodulin alfa (TM-a) for DIC and suspected DIC. Hypofibrinogenemia was defined by a fibrinogen level $<1.5 \mathrm{~g} / \mathrm{L}$.

Results: Hypofibrinogenemia was observed in $10.3 \%$ of patients with infectious diseases. The frequencies of both bleeding and organ failure symptoms, and the scores for organ failure or the DIC diagnostic criteria were significantly higher in infectious disease patients with hypofibrinogenemia, suggesting that in patients with infectious diseases, hypofibrinogenemia is associated with more progressive and severe DIC. Although the 28-day survival rate and the DIC resolution rate were both significantly lower for infectious disease patients with DIC with hypofibrinogenemia than for those without hypofibrinogenemia, this difference was not observed in DIC patients with hematological diseases.

Conclusions: Hypofibrinogenemia among infectious disease patients with DIC may reflect increased consumption of fibrinogen due to accelerated coagulation reactions, while hypofibrinogenemia among hematological disease patients with DIC may be caused by fibrinogenolysis due to hyperfibrinolysis, and frequently results in bleeding and multipleorgan failure.
\end{abstract}

Keywords: Disseminated intravascular coagulation, Hypofibrinogenemia, Thrombomodulin, Post-marketing surveillance, Infectious disease, Mortality

\footnotetext{
* Correspondence: wadahide@clin.medic.mie-u.ac.jp

${ }^{2}$ Department of General Medicine, Mie Prefectural General Medical Center, Yokkaichi, Japan

Full list of author information is available at the end of the article
}

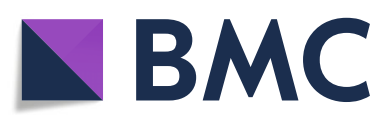

(- The Author(s). 2021 Open Access This article is licensed under a Creative Commons Attribution 4.0 International License, which permits use, sharing, adaptation, distribution and reproduction in any medium or format, as long as you give appropriate credit to the original author(s) and the source, provide a link to the Creative Commons licence, and indicate if changes were made. The images or other third party material in this article are included in the article's Creative Commons licence, unless indicated otherwise in a credit line to the material. If material is not included in the article's Creative Commons licence and your intended use is not permitted by statutory regulation or exceeds the permitted use, you will need to obtain permission directly from the copyright holder. To view a copy of this licence, visit http://creativecommons.org/licenses/by/4.0/. The Creative Commons Public Domain Dedication waiver (http://creativecommons.org/publicdomain/zero/1.0/) applies to the data made available in this article, unless otherwise stated in a credit line to the data. 


\section{Introduction}

Disseminated intravascular coagulation (DIC), which occurs in association with various underlying diseases, such as sepsis, hematological malignancy, solid tumors and aneurysm, is often associated with severe and lifethreatening bleeding or organ failure with [1-3]. DIC is characterized by the systemic activation or consumption of components in the coagulation pathways, such as increased fibrin generation or thrombocytopenia, resulting in multiple-organ failure or bleeding tendency $[4,5]$.

The diagnostic criteria for DIC have been established by the Japanese Ministry of Health and Welfare (JMHW) [6], the International Society of Thrombosis Haemostasis (ISTH) [4], the Japanese Association for Acute Medicine (JAAM) [7] and the Japanese Society of Thrombosis and Hemostasis (JSTH) [8]. These four sets of diagnostic criteria are all based on scoring systems using similar laboratory tests; however, the cutoff values for DIC scores and laboratory test results differ. The JSTH diagnostic criteria $[8,9]$ classified the diseases underlying DIC into infectious, hematopoietic disorder and basic types.

The guidelines for the diagnosis and treatment of DIC have been published by the British Committee for Standards in Haematology (BCSH) [10], the JSTH [11], the Italian Society for Thrombosis and Haemostasis (SISET) [12] and the ISTH [13]. Most guidelines show that infectious-type DIC is frequently associated with organ failure, whereas hypofibrinogenemia or bleeding tendency is less common. They have recommended the treatment of DIC via treatment of the underlying diseases, along with supportive therapy such as administration of platelet concentrates and fresh frozen plasma [10-13]. The administration of antithrombin (AT) and thrombomodulin alfa (TM- $\alpha)$ are also recommended in the JSTH guidelines [11, 14]. A phase 3 study [15] and several retrospective studies [16-18], including postmarketing surveillance (PMS), have reported the efficacy and safety of TM- $\alpha$ for DIC patients with infectious and hematological diseases. A randomized controlled trial of AT, activated protein $\mathrm{C}$ and $\mathrm{TM}-\alpha$ for severe sepsis failed to confirm any significant improvement in the outcomes of patients with severe sepsis [19-21].

The present study examined the frequency of hypofibrinogenemia in infectious and hematological diseases with DIC and suspected DIC, and analyzed the characteristics of hypofibrinogenemia in infectious disease patients with DIC.

\section{Patients and methods}

The clinical characteristics and treatment outcomes of patients with DIC and suspected DIC that was treated with TM- $\alpha$ between May 2008 and April 2010 were retrospectively analyzed in a subgroup analysis of PMS data. The original PMS study was an open-label, multicenter, non-interventional, prospective, observational cohort study of patients with DIC who received TM- $\alpha$ [16]. The PMS for TM- $\alpha$ was conducted in accordance with the Japanese Society on Thrombosis and Hemostasis Post-Marketing Surveillance Committee for Recomodulin Injection and the guidelines for Good Postmarketing Study Practice, as required by the Japanese Ministry of Health, Labour, and Welfare (JMHLW). We used existing data without personally identifiable information throughout our study. The original PMS study was therefore exempted from local institutional review and formal approval, as well as the requirement for informed consent. In the PMS for TM- $\alpha$, all patients were diagnosed with DIC and suspected DIC by attending physicians based on JMHLW or JAAM diagnostic criteria.

The PMS data included 3204 patients with DIC and suspected DIC (patients with infectious diseases, $n=2083$; patients with hematological diseases, $n=1121$ ). The underlying diseases of the patients with infectious diseases were as follows; sepsis $(n=837)$, pneumonia $(n=359)$, peritonitis $(n=260)$, urinary tract infection $(n=163)$, hepatobiliary pancreatic system infection $(n=117)$, skin and muscle infections $(n=55)$, gastrointestinal infections $(n=53)$, meningitis $(n=37)$, viral infection $(n=25)$, wound infection $(n=24)$, pleural infection $(n=18)$, and others $(n=135)$. The underlying diseases of the patients with hematological diseases were as follows; acute myeloid leukemia $(n=235)$, acute promyelocytic leukemia $(n=$ $167)$, non-Hodgkin's lymphoma $(n=156)$, acute lymphocytic leukemia $(n=144)$, acute myelomonocytic leukemia $(n=55)$, myelodysplastic syndromes $(n=51)$, acute monocytic leukemia $(n=47)$, multiple myeloma $(n=35)$, adult T cell leukemia $(n=18)$, hematopoietic stem cell transplantation $(n=17)$, chronic myelogenous leukemia $(\mathrm{n}=$ $17)$, hemophagocytic syndrome $(n=14)$, acute erythroid leukemia $(n=13)$, febrile neutropenia $(n=11)$, acute megakaryoblastic leukemia $(n=7)$, chronic lymphocytic leukemia $(\mathrm{n}=7)$, aplastic anemia $(n=5)$, Hodgkin's lymphoma $(n=5)$, and others $(n=117)$.

The DIC resolution rate, 28-day survival rate, rates of adverse drug reaction and bleeding-related adverse drug reactions were investigated. The degree of coagulopathy was evaluated by calculating DIC scores according to the DIC diagnostic criteria of the JAAM [7] for infectious diseases and those of the JMHW [6] and ISTH [4] for hematological diseases. After treatment with TM- $\alpha$, resolution of DIC was defined as a score $\leq 3$ using the diagnostic criteria of the JAAM, $\leq 2$ using the diagnostic criteria of the JMHW for DIC in patients with hematological diseases, $\leq 5$ using the diagnostic criteria of the JMHW for infectious diseases, and $\leq 4$ using the diagnostic criteria of the ISTH for both diseases. The PMS for TM- $\alpha$ started before the establishment of the 
JSTH DIC diagnostic criteria. As a result, there were few records that included "change of platelet count" or a few data for soluble fibrin and thrombin-antithrombin complex (TAT); thus, the JSTH scores could not be calculated. Survival was calculated at 28 days from the beginning of TM- $\alpha$ treatment or at the end of observation.

In patients with infectious diseases, the severity of organ failure was assessed using the sequential organ failure assessment (SOFA) score [22]. The symptoms of organ failure were decided by the attending physician based on clinical signs indicating organ dysfunction due to DIC [6]. Laboratory tests such as the white blood cell (WBC) count and platelet count, and the measurement of hemoglobin, albumin, lactate dehydrogenase (LDH), total bilirubin (T-Bil), creatine and C-reactive protein (CRP) levels, and hemostatic tests such as the prothrombin time (PT)-international ratio (INR), activated partial thromboplastin time (APTT), fibrinogen, fibrinogen, fibrin degradation products (FDP), D-dimer, AT, protein $\mathrm{C}$, TAT and plasmin- $\alpha 2$ plasmin inhibitor complex (PIC) were performed in each participating institute. Patients with fibrinogen $<1.5 \mathrm{~g} / \mathrm{L}$ were considered to have hypofibrinogenemia. The rates of adverse drug reaction and bleeding-related adverse drug reaction were evaluated from the start of TM- $\alpha$ treatment to day 28 after the end of TM- $\alpha$ treatment. The safety data were coded using preferred terms from the Japanese translation of the Medical Dictionary for Regulatory Activities (version 13.1).

\section{Statistical analysis}

In the descriptive analyses of baseline characteristics, numerical data were expressed as the median (Q1, Q3; interquartile range). The chi-square test and the Wilcoxon signed-rank test were performed as statistical analyses. $P$ values of $<0.05$ were considered to indicate statistical significance. Multiplicity adjustment was not considered. All analyses were performed using SAS version 9.4 (SAS Institute, Cary, NC) by EPS Corporation (Tokyo, Japan) according to the plan for the statistical analysis.

\section{Results}

Among cases in which the fibrinogen levels were measured, hypofibrinogenemia (fibrinogen $<1.5 \mathrm{~g} / \mathrm{L}$ ) was observed in $10.3 \%(215 / 2083)$ of the patients with infectious diseases, and $27.8 \%(312 / 1121)$ of the patients with hematological diseases (Table 1). In both diseases, the ratio of males was slightly lower among patients with hypofibrinogenemia than among those without hypofibrinogenemia. Before registration, the administration rate of AT concentrate administration was significantly lower in the infectious disease patients with hypofibrinogenemia than in those without

Table 1 Demographic characteristics in infectious disease and hematological disease patients with or without hypofibrinogenemia

\begin{tabular}{|c|c|c|c|c|c|c|c|c|c|c|}
\hline \multirow[b]{2}{*}{ Fibrinogen, $g / L$} & \multicolumn{5}{|c|}{ Infectious disease } & \multicolumn{5}{|c|}{ Hematological disease } \\
\hline & $\geq 1.5$ & & $<1.5$ & & $p$-value & $\geq 1.5$ & & $<1.5$ & & $p$-value \\
\hline Age, years & 1865 & $71(60-79)$ & 215 & $70(53-79)$ & 0.1094 & 809 & $61(43-70)$ & 311 & $63(41-73)$ & 0.0578 \\
\hline Male & 1868 & $1096(58.7)$ & 215 & $109(50.7)$ & 0.0249 & 809 & $506(62.5)$ & 312 & $165(52.9)$ & 0.0045 \\
\hline Body weight, kg & 1861 & $50(40-60)$ & 215 & $53(45-62)$ & 0.0005 & 808. & $55(48.4-64)$ & 312 & $55(48.2-63.8)$ & 0.7995 \\
\hline SOFA score & 1164 & $10(7-13)$ & 118 & $11(8-15)$ & 0.0144 & - & - & - & - & - \\
\hline JMHW DIC score & 1081 & $6(5-7)$ & 104 & $9(7-10)$ & $<0.0001$ & 551 & $4(3-5)$ & 226 & $6(5-7)$ & $<0.0001$ \\
\hline ISTH DIC score & 580 & $4(4-5)$ & 54 & $5(4-7)$ & 0.0002 & 209 & $5(4-6)$ & 81 & $6(5-7)$ & $<0.0001$ \\
\hline JAAM DIC score & 1198 & $5(4-7)$ & 119 & $6(5-8)$ & 0.0005 & - & - & - & - & - \\
\hline Pre-administration & 1868 & & 215 & & & 809 & & 312 & & \\
\hline AT concentrate & & $364(19.5)$ & & 27 (12.6) & 0.0138 & & $62(7.7)$ & & $13(4.2)$ & 0.0357 \\
\hline Gabexate mesylate & & $343(18.4)$ & & $42(19.5)$ & 0.6748 & & $61(7.5)$ & & $29(9.3)$ & 0.3326 \\
\hline Nafamostat mesylate & & $175(9.4)$ & & $12(5.6)$ & 0.0658 & & $29(3.4)$ & & $12(3.8)$ & 0.8344 \\
\hline Unfractionated heparin & & $91(4.9)$ & & $6(2.8)$ & 0.1703 & & $29(2.4)$ & & $7(2.2)$ & 0.2537 \\
\hline LMWH & & $34(1.8)$ & & $5(2.3)$ & 0.6046 & & $65(8.0)$ & & $10(3.2)$ & 0.0037 \\
\hline Danaparoid sodium & & $66(3.5)$ & & $8(3.7)$ & 0.8880 & & $31(3.8)$ & & $20(6.4)$ & 0.0634 \\
\hline Comorbidities & 1868 & & 215 & & & 809 & & 312 & & \\
\hline Bleeding symptoms & & $234(12.5)$ & & $54(25.1)$ & $<0.0001$ & & $225(27.8)$ & & $144(46.2)$ & $<0.0001$ \\
\hline Organ symptoms & & $861(46.1)$ & & $116(54.0)$ & 0.0292 & & $221(27.3)$ & & $71(22.8)$ & 0.1189 \\
\hline
\end{tabular}

Data are given as $\mathrm{n}(\%)$ or median (IQR)

DIC, disseminated intravascular coagulation; AT, antithrombin; LMWH, low molecular weight heparin; SOFA, sequential organ failure assessment;

JMHW, Japanese Ministry of Health and Welfare; ISTH, International Society of Thrombosis Haemostasis; JAAM, Japanese Association for Acute Medicine 
hypofibrinogenemia. The rates of bleeding and organ failure symptoms were both significantly higher in infectious disease patients with hypofibrinogenemia than in those without hypofibrinogenemia. The median SOFA, JMHW, ISTH and JAAM scores were significantly higher in infectious disease patients with hypofibrinogenemia $(11,9,5$ and 6 , respectively) than in those without hypofibrinogenemia $(10,6,4$ and 5 , respectively; Table 1 ).

Regarding the hemostatic examinations, the PT-INR was significantly prolonged among patients with hypofibrinogenemia, and the fibrinogen and AT levels were significantly lower among patients with hypofibrinogenemia than among those without hypofibrinogenemia (Supplementary Table 1). The FDP, D-dimer and TAT levels were significantly higher among patients with hypofibrinogenemia than among those without hypofibrinogenemia. The PIC levels in infectious disease patients with and without hypofibrinogenemia did not differ to a statistically significant extent; however, the PIC levels in hematological disease patients with hypofibrinogenemia were significantly higher than in those without hypofibrinogenemia. The platelet counts in infectious disease patients were significantly lower among patients with hypofibrinogenemia than among those without hypofibrinogenemia (Supplementary Table 2). In both diseases, the albumin and LDH levels were significantly higher and the CRP levels were significantly lower in patients with hypofibrinogenemia than in those without hypofibrinogenemia.

In the treatment of DIC, the TM- $\alpha$ dose and the period of administration in patients with and without hypofibrinogenemia did not differ to a statistically significant extent (Supplementary Table 3). AT concentrate, gabexate mesylate, nafamostat mesylate, unfractionated heparin, low molecular weight heparin, danaparoid sodium, platelet concentrates, fresh frozen plasma and red blood cells were also administered, as shown in Supplementary Table 3. Although the 28-day survival rate of infectious disease patients with hypofibrinogenemia (50.0\%) was significantly lower $(p<0.0001)$ than that of infectious disease patients without hypofibrinogenemia (71.6\%), the 28 -day survival rate of hematological diseases patients with and without hypofibrinogenemia did not differ to a statistically significant extent (Table 2). The resolution rates according to the JMHW, ISTH and JAAM DIC diagnostic criteria were significantly lower $(p=0.0058, p<0.0001$ and $\mathrm{p}<0.0001$, respectively) in infectious disease patients with hypofibrinogenemia than in those without hypofibrinogenemia; this difference was not observed in the hematological disease patients with and without hypofibrinogenemia. In both the infectious disease and hematological disease groups, the clinical course of bleeding symptoms did not differ between patients with and without hypofibrinogenemia.

\section{Discussion}

As hypofibrinogenemia is frequently related to the bleeding tendency in DIC patients with hematological disease and organ failure is often associated with DIC patients with infectious disease, hypofibrinogenemia is considered to be frequently associated with DIC in patients with hematological disease, but rarely with DIC in patients with infectious disease $[5,14]$. However, some septic cases have been reported to be associated with severe bleeding [23, 24]. Our analysis of the PMS data revealed that hypofibrinogenemia was present in $10.3 \%$ of infectious diseases with DIC and suspected DIC, suggesting that hypofibrinogenemia is sometimes associated with DIC in infectious diseases. As the DIC scores were high, many patients in this study were considered to meet the definition of severe DIC. Thus, the frequency of hypofibrinogenemia in DIC patients with infectious disease may generally be less than $10.3 \%$. Both the frequency of bleeding and organ failure symptoms, and SOFA, JMHW, ISTH and JAAM scores in infectious disease were significantly higher in DIC patients with hypofibrinogenemia than in those without hypofibrinogenemia. This suggests that hypofibrinogenemia in DIC patients with infectious disease can be considered to

Table 2 Outcomes of infectious disease or hematological disease patients in with or without hypofibrinogenemia

\begin{tabular}{|c|c|c|c|c|c|c|c|c|c|c|}
\hline \multirow[b]{2}{*}{ Fibrinogen, $g / L$} & \multicolumn{5}{|c|}{ Infectious diseases } & \multicolumn{5}{|c|}{ Hematological diseases } \\
\hline & \multicolumn{2}{|l|}{$\geq 1.5$} & \multicolumn{2}{|c|}{$<1.5$} & \multirow{2}{*}{$\begin{array}{c}\text { p-value } \\
<0.0001\end{array}$} & \multicolumn{2}{|l|}{$\geq 1.5$} & \multicolumn{2}{|l|}{$<1.5$} & \multirow{2}{*}{$\begin{array}{c}\text { p-value } \\
0.4566\end{array}$} \\
\hline 28-day survival & 1846 & $1321(71.6)$ & 212 & $106(50.0)$ & & 807 & $587(72.7)$ & 312 & $220(70.5)$ & \\
\hline JMHW DIC resolution & 683 & $427(62.5)$ & 98 & $47(48.0)$ & 0.0058 & 476 & $275(57.8)$ & 231 & $113(48.9)$ & 0.0265 \\
\hline ISTH DIC resolution & 651 & $449(69.0)$ & 119 & $57(47.9)$ & $<0.0001$ & 385 & $240(62.3)$ & 202 & $116(57.4)$ & 0.2472 \\
\hline JAAM DIC resolution & 1266 & $513(40.5)$ & 156 & $30(19.2)$ & $<0.000$ & - & - & - & - & \\
\hline \multicolumn{11}{|c|}{ Clinical course of bleeding symptoms } \\
\hline Disappeared+Improved & 216 & $99(45.8)$ & 48 & $24(50.0)$ & 0.6007 & 217 & $110(50.7)$ & 142 & $86(60.6)$ & 0.0662 \\
\hline Unchanged+Exacerbated & 216 & $117(54.2)$ & 48 & $24(50.0)$ & & 217 & 107 (49.3) & 142 & $56(39.4)$ & \\
\hline
\end{tabular}

Data are given as $\mathrm{n}(\%)$

DIC, disseminated intravascular coagulation; JMHW, Japanese Ministry of Health and Welfare; ISTH, International Society of Thrombosis Haemostasis;

JAAM, Japanese Association for Acute Medicine 
indicate severe and advanced DIC, in which patients exhibit both bleeding and organ-failure symptoms.

The platelet count in infectious diseases was lower in DIC patients with hypofibrinogenemia than in those without hypofibrinogenemia, suggesting that more severe thrombocytopenia contributes to increased bleeding symptoms or is reflected by multiple thrombi and a subsequent poor prognosis.

The APTT, AT, FDP, D-dimer and TAT levels were significantly greater in patients with hypofibrinogenemia than in those without. In DIC patients with infectious disease, no significant differences in PIC levels were seen between the subgroups with and without hypofibrinogenemia. However, in hematological disease patients with DIC, the PIC levels were significantly higher in patients with hypofibrinogenemia than in those without. These findings indicate that the mechanisms of hypofibrinogenemia in DIC may differ between patients with infectious diseases and hematological diseases, in whom DIC often causes hyperfibrinolysis due to hyperplasminogen activation by leukemic cells $[25,26]$. Conversely, the relatively low levels of PIC in infectious disease did not show hyperfibrinolysis in DIC patients with hypofibrinogenemia, and the increased FDP and Ddimer levels showed the progression of DIC, resulting in high SOFA scores in patients with this type of DIC.

Reduced AT levels may be caused by decreased production due to liver dysfunction, the consumption of $\mathrm{AT}$, leakage into the third space due to increased permeability of vascular endothelial cells [27] or less administration of AT. Although a high DIC score indicates the existence of consumption coagulopathy in DIC patients with severe AT deficiency, decreased albumin and cholinesterase levels and increased T-Bil and creatinine levels suggest that liver dysfunction, plasma leakage, or renal dysfunction might play some role in AT deficiency in patients with DIC. In comparison, the mechanisms underlying hypofibrinogenemia in patients with infectious diseases and DIC are suggested to involve a progressive hypercoagulable state that disseminates and causes the consumption of coagulation factors, such as fibrinogen.

Before registration, the frequency of AT administration was significantly lower in infectious disease patients with hypofibrinogenemia than in those without, suggesting that single anticoagulation therapy tended to be selected in daily clinical practice. Another possibility is that DIC in patients with infectious diseases may result in hypofibrinogenemia with low AT levels in patients who are not treated with AT. The effect of recombinant thrombomodulin administration in sepsis-induced coagulopathy in the SCARLET study [21] was not statistically significant. However, a post hoc analysis revealed a 5.4\% reduction in absolute mortality among patients who fulfilled the entry criterion at baseline [28]. Although no significant differences in the TM- $\alpha$ dose or the period of administration were seen between these two states in both types of DIC, the 28-day survival rate and resolution rate according to JMHW, ISTH or JAAM diagnostic criteria in infectious diseases were significantly lower in DIC patients with hypofibrinogenemia than in DIC patients without hypofibrinogenemia. This was only observed in DIC patients with infectious disease. As the mechanism of hypofibrinogenemia in DIC differs between infectious and hematological diseases, hypofibrinogenemia in DIC is considered to be associated with a high risk of infectious diseases, but not hematological diseases. That is, hypofibrinogenemia in infectious diseases may correlate with the severity of DIC, but may be caused by hyperfibrinolysis in hematological diseases, and thus is not associated with poor outcomes. Conversely, no significant differences in the 28-day survival rate or the resolution rate (according to the JMHW, ISTH or JAAM diagnostic criteria for DIC) were evident in hematological disease patients with DIC. DIC patients with increased fibrinogen levels were previously reported to show poor outcomes; however, that previous study included many patients with hematological diseases [29]. High fibrinogen levels might be associated with a hypofibrinolytic state and elevated levels of plasminogen activator inhibitor 1 [30], which is a biomarker for a poor outcome or organ failure due to infection. Production of fibrinogen is upregulated by the liver in infectious diseases, resulting in the observation of increased fibrinogen levels in many DIC patients with infectious diseases [3]. However, hypofibrinogenemia is sometimes caused by consumption of fibrinogen under conditions of hypercoagulability, suppressed production by liver injury, or hyperfibrinolysis [3]. After improvement of these conditions, the plasma fibrinogen level will increase and show various levels depending on the clinical course of infectious disease or liver function. Hypofibrinogen levels may thus strongly reflect the clinical course of the underlying disease, liver function, fibrinolysis, or timing of evaluations [5]. PMS data for TM- $\alpha$ did not show details of bleeding or organ failure symptoms related to the mortality in infectious disease patients with hypofibrinogenemia. Further studies to examine the relationships between severe bleeding or organ failure with hypofibrinogenemia and poor outcome in infectious disease patients with DIC are needed.

In conclusion, DIC in infectious diseases is sometimes associated with hypofibrinogenemia, which reflects the increased consumption of fibrinogen due to accelerated coagulation. Hypofibrinogenemia in DIC patients with 
infectious diseases is therefore associated with poor outcomes, and the monitoring of fibrinogen levels appears important in the management of DIC in infectious diseases.

\section{Supplementary Information}

The online version contains supplementary material available at https://doi. org/10.1186/s12959-021-00264-z.

\section{Additional file 1.}

\section{Abbreviations}

DIC: Disseminated intravascular coagulation; TM-a: Thrombomodulin alfa; JMHW: Japanese Ministry of Health and Welfare; ISTH: International Society of Thrombosis Haemostasis; JAAM: Japanese Association for Acute Medicine; JSTH: Japanese Society of Thrombosis and Hemostasis; BCSH: British Committee for Standards in Haematology; SISET: Italian Society for Thrombosis and Haemostasis; AT: Antithrombin; PMS: Post-marketing surveillance; JMHLW: Japanese Ministry of Health, Labour, and Welfare; TAT: Thrombin-antithrombin complex; SOFA: Sequential organ failure assessment; WBC: White blood cell; LDH: Lactate dehydrogenase; T-Bil: Total bilirubin; CRP: C-reactive protein; PT-INR: Prothrombin time international normalized ratio; APTT: Activated partial thromboplastin time; FDP: Fibrin and fibrinogen degradation products; PIC: Plasmin-a2 plasmin inhibitor complex

\section{Acknowledgements}

The authors would like to thank all investigators who participated in this PMS study.

\section{Authors' contributions}

H.W. planned the study design, interpreted the data and wrote the manuscript. H.W. oversaw the current study and supervised the co-authors. T.U., K.K., S.M., N.T., K.S., Y.S., T.I. ., T.I ${ }^{k}$, and K.O. provided advice on the study design and interpretation of the data, and reviewed the manuscript. G.H. planned the study design. The author(s) read and approved the final manuscript.

\section{Funding}

This work was supported by Asahi Kasei Pharma Corporation, which funded the analysis and English editing of the manuscript.

\section{Availability of data and materials}

The data supporting the findings of this study are available from Asahi Kasei Pharma Corporation; however, restrictions apply to the availability of these data, which were used under license for the current study, and so are not publicly available.

\section{Ethics approval and consent to participate}

Approval for this study was obtained from the appropriate ethics committees and institutional review boards of Mie Prefectural General Medical Center.

\section{Consent for publication}

Not applicable.

\section{Competing interests}

Asahi Kasei Pharma Corporation absorbed expenses for the analysis and the English language editing of manuscript. N.K. received no grants or personal fees. H.W. received grants and personal fees from Asahi Kasei Pharma Corporation and Japan Blood Products Organization outside the present work. T.U., S.M., N.T., K.S., Y.S., and K.O. received personal fees from Asahi Kasei Pharma Corporation outside the present work. K.K. and T.I. received grants and personal fees from Asahi Kasei Pharma Corporation outside the present work. G.H. is an employee of Asahi Kasei Pharma Corporation. T.I ${ }^{k}$. received research grants from Japan Blood Products Organization and Japan Immunoresearch Laboratories outside the present work.

\section{Author details}

${ }^{1}$ Faculty of Medical Technology, Teikyo University, Tokyo, Japan. ${ }^{2}$ Department of General Medicine, Mie Prefectural General Medical Center, Yokkaichi, Japan. ${ }^{3}$ Department of Medical Affairs, Asahi Kasei Pharma Corporation, Tokyo, Japan. ${ }^{4}$ Department of Internal Medicine, Miyazaki Prefectural Miyazaki Hospital, Miyazaki, Japan. ${ }^{5}$ Department of Laboratory Medicine, National Hospital Organization Takasaki General Medical Center, Takasaki, Japan. ${ }^{6}$ Department of Clinical Laboratory Medicine, Tokyo Saiseikai Central Hospital, Tokyo, Japan. ${ }^{7}$ Department of Hematology, National Hospital Organization Disaster Medical Center, Tokyo, Japan. ${ }^{8}$ Emergency and Critical Care Center, Mie University Hospital, Tsu, Japan. ${ }^{9}$ Department of Hematology, Uonuma Institute of Community Medicine, Niigata University Medical and Dental Hospital, Niigata, Japan. ${ }^{10}$ Department of Hematology, Fukushima Medical University, Fukushima, Japan. ${ }^{11}$ Department of Emergency and Disaster Medicine, Juntendo University Graduate School of Medicine, Tokyo, Japan. ${ }^{12}$ Department of Surgery, Center for Gastroenterology and Liver Disease, Kitakyushu City Yahata Hospital, Fukuoka, Japan.

Received: 1 October 2020 Accepted: 16 February 2021

Published online: 25 February 2021

\section{References}

1. Levi M, Ten Cate H. Disseminated intravascular coagulation. N Engl J Med. 1999;341:586-92.

2. Gando S, Levi M, Toh CH. Disseminated intravascular coagulation. Nat Rev Dis Primers. 2016:2:16037.

3. Wada H, Matsumoto T, Yamashita Y, Hatada T. Disseminated intravascular coagulation: testing and diagnosis. Clin Chim Acta. 2014;436C:130-4.

4. Taylor FB Jr, Toh CH, Hoots K, Wada H, Levi M. Towards a definition, clinical and laboratory criteria, and a scoring system for disseminated intravascular coagulation. Thromb Haemost. 2001;86(5):1327-30.

5. Wada H, Matsumoto T, Yamashita Y. Diagnosis and treatment of disseminated intravascular coagulation (DIC) according to four DIC guidelines. J Intensive Care. 2014;2:15

6. Kobayashi N, Maegawa T, Takada M, Tanaka H, Gonmori H. Criteria for diagnosis of DIC based on the analysis of clinical and laboratory findings in 345 DIC patients collected by the research committee on DIC in Japan. Bibl Haemotol. 1983;49:265-75.

7. Gando S, Iba T, Eguchi Y, Ohtomo Y, Okamoto K, Koseki K, et al. Japanese Association for Acute Medicine Disseminated Intravascular Coagulation (JAAM DIC) Study Group. A multicenter, prospective validation of disseminated intravascular coagulation diagnostic criteria for critically ill patients: comparing current criteria. Crit Care Med. 2006;34:625-31.

8. Asakura H, Takahashi H, Uchiyama T, Eguchi Y, Okamoto K, Kawasugi K, et al. DIC subcommittee of the Japanese society on thrombosis and hemostasis. Proposal for new diagnostic criteria for DIC from the Japanese Society on Thrombosis and Hemostasis. Thromb J. 2016;14:42.

9. Wada H, Takahashi H, Uchiyama T, Equchi Y, Okamoto K, Kawasugi K, et al. DIC subcommittee of the Japanese society on thrombosis and hemostasis. The approval of revised diagnostic criteria for DIC from the Japanese Society on Thrombosis and Hemostasis. Thromb J. 2017;15:17.

10. Levi M, Toh CH, Thachil J, Watson HG. Guidelines for the diagnosis and management of disseminated intravascular coagulation. British Committee for Standards in Haematology. Br J Haematol. 2009;145:24-33.

11. Wada H, Asakura H, Okamoto K, Iba T, Uchiyama T, Kawasugi K, et al. Japanese Society of Thrombosis Hemostasis/DIC subcommittee. Japanese Society of Thrombosis Hemostasis/DIC subcommittee. Expert consensus for the treatment of disseminated intravascular coagulation in Japan. Thromb Res. 2010;125:6-11.

12. Di Nisio M, Baudo F, Cosmi B, D'Angelo A, De Gasperi A, Malato A, et al. on behalf of the Italian Society for Thrombosis and Haemostasis. Diagnosis and treatment of disseminated intravascular coagulation: guidelines of the Italian Society for Haemostasis and Thrombosis (SISET). Thromb Res. 2012; 129:e177-84

13. Wada H, Thachil J, Di Nisio M, Mathew P, Kurosawa S, Gando S, et al. The scientific standardization committee on DIC of the international society on thrombosis Haemostasis. Guidance for diagnosis and treatment of DIC from harmonization of the recommendations from three guidelines. J Thromb Haemost. 2013;11:761-7.

14. Wada H, Okamoto K, Iba T, Kushimoto S, Kawasugi K, Gando S, et al. Japanese Society of Thrombosis Hemostasis/DIC subcommittee. Addition of 
recommendations for the use of recombinant human thrombomodulin to the "Expert consensus for the treatment of disseminated intravascular coagulation in Japan". Thromb Res. 2014;134:924-5.

15. Saito H, Maruyama I, Shimazaki S, Yamamoto Y, Aikawa N, Ohno R, et al. Efficacy and safety of recombinant human soluble thrombomodulin (ART123) in disseminated intravascular coagulation: results of a phase III, randomized, double-blind clinical trial. J Thromb Haemost. 2007;5:31-41.

16. Mimuro J, Takahashi H, Kitajima I, Tsuji H, Eguchi Y, Matsushita T, et al. Impact of recombinant soluble thrombomodulin (thrombomodulin alfa) on disseminated intravascular coagulation. Thromb Res. 2013;131:436-43.

17. Asakura $H$, Takahashi $H$, Tsuji H, Matsushita T, Ninomiya $H$, Honda $G$, et al. Post-marketing surveillance of thrombomodulin alfa, a novel treatment of disseminated intravascular coagulation - safety and efficacy in 1,032 patients with hematologic malignancy. Thromb Res. 2014;133:364-70.

18. Eguchi Y, Gando S, Ishikura H, Saitoh D, Mimuro J, Takahashi H, et al. Postmarketing surveillance data of thrombomodulin alfa: sub-analysis in patients with sepsis-induced disseminated intravascular coagulation. J Intensive Care. 2014;2:30.

19. Warren BL, Eid A, Singer P, Pillay SS, Carl P, Novak I, et al. Caring for the critically ill patient. High-dose antithrombin III in severe sepsis: a randomized controlled trial. JAMA. 2001;286:1869-78.

20. Ranieri VM, Thompson BT, Barie PS, Dhainaut JF, Douglas IS, Finfer S, et al PROWESS-SHOCK study group. Drotrecogin alfa (activated) in adults with septic shock. N Engl J Med. 2012;366:2055-64.

21. Vincent JL, Francois B, Zabolotskikh I, Daga MK, Lascarrou JB, Kirov MY, et al. SCARLET Trial Group. Effect of a recombinant human soluble thrombomodulin on mortality in patients with sepsis-associated coagulopathy: the SCARLET randomized clinical trial. JAMA. 2019;321:19932002.

22. Vincent JL, Moreno R, Takala J, Willatts S, De Mendonça A, Bruining H, et al. The SOFA (Sepsis-related organ failure assessment) score to describe organ dysfunction/failure. On behalf of the working group on Sepsis-related problems of the European Society of Intensive Care Medicine. Intensive Care Med. 1996;22:707-10.

23. Okuzono S, Ishimura M, Kanno S, Sonoda M, Kaku N, Motomura Y, et al. Streptococcus pyogenes-purpura fulminans as an invasive form of group a streptococcal infection. Ann Clin Microbiol Antimicrob. 2018;17:31.

24. Iba T, Gando S, Saitoh D, keda T, Anan H, Oda S, et al. Efficacy and bleeding risk of antithrombin supplementation in patients with septic disseminated intravascular coagulation: a third survey. Clin Appl Thromb Hemost. 2017;23: 422-8.

25. Wada H, Nagano T, Tomeoku M, Kuto M, Karitani Y, Deguchi K, et al. Coagulant and fibrinolytic activities in the leukemic cell lysates. Thromb Res. 1982:30:315-22.

26. Wada H, Kumeda Y, Ogasawara Z, Ohiwa M, Kaneko T, Tamaki S, et al. Plasminogen activators and their inhibitors in leukemic cell homogenates. Am J Hematol. 1993;42:166-70.

27. Wada H. Disseminated intravascular coagulation. Clin Chim Acta. 2004;344: 13-21.

28. Ito T, Thachil J, Asakura H, Levy JH, Iba T. Thrombomodulin in disseminated intravascular coagulation and other critical conditions-a multi-faceted anticoagulant protein with therapeutic potential. Crit Care. 2019;23:280.

29. Wada H, Mori Y, Sakakura M, Gabazza EC, Kushiya F, Watanabe M, et al. High plasma levels of fibrinogen are associated with poor outcome. Am J Hematol. 2003;72:1-7.

30. Watanabe R, Wada H, Miura Y, Murata Y, Watanabe Y, Sakakura M, et al. Plasma levels of total plasminogen activator inhibitor-I and tissue plasminogen activator/inhibitor complex in patients with disseminated intravascular coagulation and thrombotic thrombocytopenic purpura. Clin Appl Thromb Haemost. 2001;7:229-33.

\section{Publisher's Note}

Springer Nature remains neutral with regard to jurisdictional claims in published maps and institutional affiliations.

Ready to submit your research? Choose BMC and benefit from:

- fast, convenient online submission

- thorough peer review by experienced researchers in your field

- rapid publication on acceptance

- support for research data, including large and complex data types

- gold Open Access which fosters wider collaboration and increased citations

- maximum visibility for your research: over $100 \mathrm{M}$ website views per year

At BMC, research is always in progress.

Learn more biomedcentral.com/submissions 